\title{
A Simple $n$-Dimensional Intrinsically Universal Quantum Cellular Automaton
}

\author{
Pablo Arrighi and Jonathan Grattage \\ ${ }^{1}$ University of Grenoble, Laboratoire LIG, \\ Bâtiment IMAG C, 220 rue de la Chimie, \\ 38400 Saint-Martin-d'Hères, France \\ 2 Ecole Normale Supérieure de Lyon, Laboratoire LIP, \\ 46 allée d'Italie, 69364 Lyon cedex 07, France
}

\begin{abstract}
We describe a simple $n$-dimensional quantum cellular automaton (QCA) capable of simulating all others, in that the initial configuration and the forward evolution of any n-dimensional QCA can be encoded within the initial configuration of the intrinsically universal QCA. Several steps of the intrinsically universal QCA then correspond to one step of the simulated QCA. The simulation preserves the topology in the sense that each cell of the simulated QCA is encoded as a group of adjacent cells in the universal QCA.
\end{abstract}

\section{Introduction}

Cellular automata (CA), first introduced by Von Neumann [36, consist of an array of identical cells, each of which may take one of a finite number of possible states. The whole array evolves in discrete time steps by iterating a function $G$. This global evolution $G$ is shift-invariant (it acts everywhere the same) and local (information cannot be transmitted faster than some fixed number of cells per time step). Because this is a physics-like model of computation [18, Feynman [16, and later Margolus [19, suggested that quantising this model was important, for two reasons: firstly, because in CA computation occurs without extraneous (unnecessary) control, hence eliminating a source of decoherence; and secondly because they are a good framework in which to study the quantum simulation of a quantum system. From a computation perspective there are other reasons to study QCA, such as studying space-sensitive problems in computer science, e.g. 'machine self-reproduction' 36 or 'Firing Squad Synchronisation', which QCA allow in the quantum setting. There is also a theoretical physics perspective, where CA are used as toy models of quantum space-time [17]. The first approach to defining QCA [2]15|37] was later superseded by a more axiomatic approach 8/9130 together with the more operational approaches 11/24 28 29 34 37.

The most well known CA is Conway's 'Game of Life', a two-dimensional CA which has been shown to be universal for computation, in the sense that any Turing Machine (TM) can be encoded within its initial state and then executed 
by evolution of the CA. Because TM have long been regarded as the best definition of 'what an algorithm is' in classical computer science, this result could have been perceived as providing a conclusion to the topic of CA universality. This was not the case, because CA do more than just running any algorithm. They run distributed algorithms in a distributed manner, model phenomena together with their spatial structure, and allow the use of the spatial parallelism inherent to the model. These features, modelled by CA and not by TM, are all interesting, and so the concept of universality must be revisited in this context to account for space. This is achieved by returning to the original meaning of the word universality [1]10]2, namely the ability for one instance of a computational model to be able to simulate other instances of the same computational model. Intrinsic universality formalises the ability of a CA to simulate another in a space-preserving manner 20[26]32], and was extended to the quantum setting in $315[6]$.

There are several related results in the CA literature. For example, refs. 18122 23 provide computation universal Reversible Partitioned CA constructions, whereas ref. 21 deals with their ability to simulate any CA in the onedimensional case. The problem of minimal intrinsically universal CA was addressed, $c f$. [27, and for Reversible CA (RCA) the issue was tackled by DurandLose 1314. The difficulty is in having an $n$-dimensional RCA simulate all other $n$-dimensional RCA and not, say, the $(n-1)$-dimensional RCA, otherwise a history-keeping dimension could be used, as by Toffoli 33. There are also several other QCA related results. Watrous [38 has proved that QCA are universal in the sense of QTM. Shepherd, Franz and Werner [31 defined a class of QCA where the scattering unitary $U_{i}$ changes at each step $i$ (CCQCA). Universality in the circuit-sense has already been achieved by Van Dam 34, Cirac and Vollbrecht [35, Nagaj and Wocjan 24] and Raussendorf 29]. In the bounded-size configurations case, circuit universality coincides with intrinsic universality, as noted by Van Dam 34. QCA intrinsic universality in the one-dimensional case is resolved in ref. 4]. Given the crucial role of this in classical CA theory, the issue of intrinsic universality in the $n$-dimensional case began to be addressed in refs. [56, where it was shown that a simple subclass of QCA, namely Partitioned QCA (PQCA), are intrinsically universal. Having shown that PQCA are intrinsically universal, it remains to be shown that there exists a $n$-dimensional PQCA capable of simulating all other $n$-dimensional PQCA for $n>1$, which is presented here.

PQCA are QCA of a particular form, where incoming information is scattered by a fixed unitary $U$ before being redistributed. Hence the problem of finding an intrinsically universal PQCA reduces to finding some universal scattering unitary $U$ (this is made formal in section 2.2, see Fig 2). Clearly the universality requirement on $U$ is much more difficult than just quantum circuit universality. This is because the simulation of a QCA $H$ has to be done in a parallel, spacepreserving manner. Moreover we must simulate not only an iteration of $H$ but several $\left(H^{2}, \ldots\right)$, so after every simulation the universal PQCA must be ready for a further iteration. 
From a computer architecture point of view, this problem can be recast in terms of finding some fundamental quantum processing unit which is capable of simulating any other network of quantum processing units, in a space-preserving manner. From a theoretical physics perspective, this amounts to specifying a scattering phenomenon that is capable of simulating any other, again in a spacepreserving manner.

\section{An Intrinsically Universal QCA}

The aim is to find a particular $U$-defined PQCA which is capable of intrinsically simulating any $V$-defined PQCA, for any $V$. In order to describe such a $U$ defined PQCA in detail, two things must be given: the dimensionality of the cells (including the meaning attached to each of the states they may take), and the way the scattering unitary $U$ acts upon these cells. First we discuss the general scheme used to solve this problem, and then describe the PQCA implementing it. The necessary definitions for $n$-dimensional QCA are given in refs. [56].

\subsection{Circuit Universality versus Intrinsic Universality in Higher Dimensions}

As already discussed, intrinsic universality refers to the ability for one CA to simulate any other $\mathrm{CA}$ in a way which preserves the spatial structure of the simulated CA. Conversely, computation universality refers to the ability of a CA to simulate any TM, and hence run any algorithm. Additionally, circuit universality is the ability of one CA to simulate any circuit. These are NAND gate circuits for classical circuits and $\mathrm{CA}$, and TOFFOLI gate circuits for reversible circuits and CA. Informally, in a quantum setting, circuit universality is the ability of a PQCA to simulate any unitary evolution expressed as a combination of a universal set of quantum gates, such as the standard gate set: CNOT, $\mathrm{R}\left(\frac{\pi}{4}\right)$ (also known as the $\frac{\pi}{8}$ gate), and the HADAMARD gate. The relationships between these three concepts of CA universality have been noted previously [12]. A computation universal CA is also a circuit universal CA, because circuits are finitary computations. Moreover, an intrinsic universal CA is also a computation universal CA, because it can simulate any CA, including computation universal CA. Hence intrinsic universality implies computation universality, which implies circuit universality.

In one-dimension this is not an equivalence. Intuitively, computation universality requires more than circuit universality, namely the ability to loop the computation, which is not trivial for CA. Similarly, intrinsic universality requires more than computation universality, such as the ability to simulate multiple communicating TM. In the classical setting there are formal results that distinguish these ideas [26].

In $n$-dimensions, it is often assumed in the classical CA literature that circuit universality implies intrinsic universality, and hence these are all equivalent [26]. 
Strictly speaking this is not true. Consider a two-dimensional CA which runs onedimensional CA in parallel. If the one-dimensional CA is circuit/computation universal, but not computation/intrinsically universal, then this is also true for the two-dimensional CA. Similarly, in the PQCA setting, the two-dimensional constructions in [28] and 29] are circuit universal but not intrinsically universal.

However, this remains a useful intuition: Indeed, CA admit a block representation, where these blocks are permutations for reversible CA, while for PQCA the blocks are unitary matrices. Thus the evolution of any (reversible/quantum) CA can be expressed as an infinite (reversible/quantum) circuit of (reversible/ quantum) gates repeating across space. If a $\mathrm{CA}$ is circuit universal, and if it is possible to wire together different circuit components in different regions of space, then the CA can simulate the block representation of any CA, and hence can simulate any CA in a way which preserves its spatial structure. It is intrinsically universal. This is the route followed next in constructing the intrinsically universal $n$-dimensional PQCA. First the construction of the 'wires', which can carry information across different regions of space, is considered. Here these are signals which can be redirected or delayed using barriers, with each signal holding a qubit of information. Secondly, the 'circuit-pieces' are constructed, by implementing quantum gates which can be combined. One and two qubit gates are implemented as obstacles to, and interactions of, these signals.

\subsection{Flattening a PQCA into Space}

In the classical CA literature it is considered enough to show that the CA implements some wires carrying signals, and some universal gates acting upon them, to prove that an $n$-dimensional $\mathrm{CA}$ is in fact intrinsically universal. Any CA can be encoded into a 'wire and gates' arrangement following the above argument, but this has never been made explicit in the literature. This section makes more precise how to flatten any PQCA in space, so that it is simulated by a PQCA which implements quantum wires and universal quantum gates. Flattening a PQCA means that the infinitely repeating, two-layered circuit is arranged in space so that at the beginning all the signals carrying qubits find themselves in circuit-pieces which implement a scattering unitary of the first layer, and then all synchronously exit and travel to circuit-pieces implementing the scattering unitary of the second layer, etc. An algorithm for performing this flattening can be provided, however the process will not be described in detail, for clarity and following the classical literature, which largely ignores this process.

The flattening process can be expressed in three steps: Firstly, the $V$-defined PQCA is expanded in space by coding each cell into a hypercube of $2^{n}$ cells. This allows enough space for the scattering unitary $V$ to be applied on nonoverlapping hypercubes of cells, illustrated in the two-dimensional case in Fig. 1. Secondly, the hypercubes where $V$ is applied must be connected with wires, as shown in Fig. 1 (right). Within these hypercubes wiring is required so that incoming signals are bunched together to undergo a circuit implementation of $V$, and are then dispatched appropriately, as shown in Fig. 2 (left). This requires both time and space expansions, with factors that depend non-trivially (but 


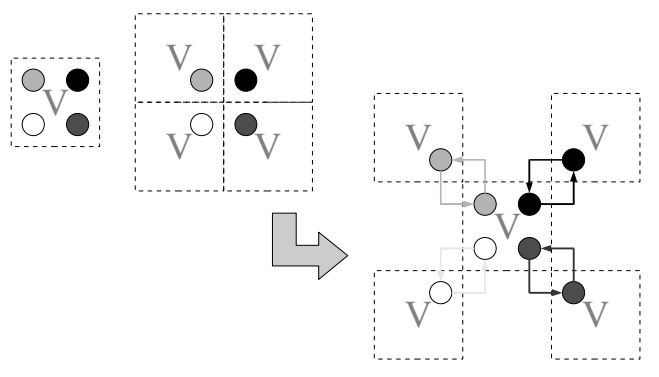

Fig. 1. Flattening a PQCA into a simulating PQCA. Left: Consider four cells (white, light grey, dark grey, black) of a PQCA having scattering unitary $V$. The first layer PQCA applies $V$ to these four cells, then the second layer applies $V$ at the four corners. Right: We need to flatten this so that the two-layers become non-overlapping. The first layer corresponds to the centre square, and the second layer to the four corner squares. At the beginning the signals (white, light grey, dark grey, black) coding for the simulated cells are in the centre square. They undergo $V$, and are directed towards the bottom left, top left, bottom right, and top right squares respectively, where they undergo $V$ but paired up with some other signals, etc.

uninterestingly) upon the size of the circuit implementation of $V$ and the way the wiring and gates work in the simulating PQCA. Next, an encoding of the circuit description of the scattering unitary $V$ is implemented in the simulating PQCA upon these incoming bunched wires, as shown in Fig. 2 (right). This completes the description of the overall scheme according to which a PQCA that is capable of implementing wires and gates is also capable of intrinsically simulating any PQCA, and hence any QCA. A particular PQCA that supports these wires and gates can now be constructed.

\subsection{Barriers and Signals Carrying Qubits}

Classical CA studies often refer to 'signals' without an explicit definition. In this context, a signal refers to the state of a cell which may move to a neighbouring cell consistently, from one step to another, by the evolution of the CA. Therefore a signal would appear as a line in the space-time diagram of the CA. These lines need to be implemented as signal redirections. A 2D solution is presented here, but this scheme can easily be extended to higher dimensions. Each cell has four possible basis states: empty $(\epsilon)$, holding a qubit signal (0 or 1$)$, or a barrier (ם). The scattering unitary $U$ of the universal PQCA acts on $2 \times 2$ cell neighbourhoods.

Signals encode qubits which can travel diagonally across the 2D space (NE, $\mathrm{SE}$, SW, or NW). Barriers do not move, while signals move in the obvious way 

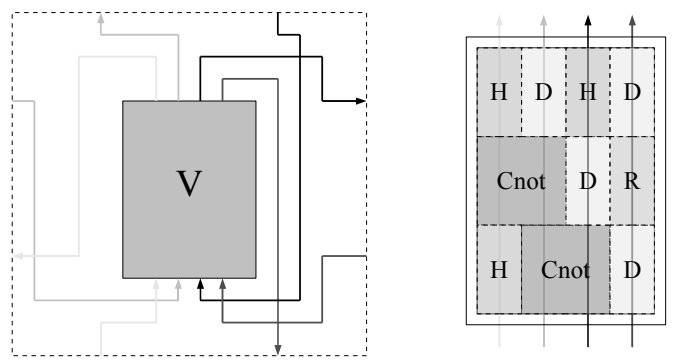

Fig. 2. Flattening a PQCA into a simulating PQCA (cont'd). Left: Within the central square the incoming signals are bunched together so as to undergo a circuit which implements $V$, and are then dispatched towards the four corners. This diagram does not make explicit a number of signal delays, which may be needed to ensure that they arrive synchronously at the beginning of the circuit implementing $V$. Right: Within the central rectangle, the circuit which implements $V$ is itself a combination of smaller circuits for implementing a universal set of quantum gates such as CNOT, HADAMARD and the $\mathrm{R}\left(\frac{\pi}{4}\right)$, together with delays. These are implemented as explained in sections 2.3 and 2.4 .

if unobstructed, as there is only one choice for any signal in any square of four cells. Hence the basic movements of signals are given by the following four rules:

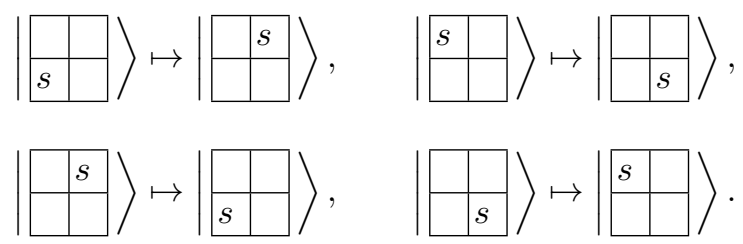

where $s \in\{0,1\}$ denotes a signal, and blank cells are empty.

The way to interpret the four above rules in terms of the scattering unitary $U$ is just case-by-case definition, i.e. they show that $U\left|\begin{array}{|l|l|}\hline s & \\ \hline s & \end{array}\right\rangle=\left|\begin{array}{|l|l}\hline & s \\ \hline & \end{array}\right\rangle$. Moreover, each rule can be obtained as a rotation of another, hence by stating that the $U$-defined PQCA is isotropic the first rule above suffices. This convention will be used throughout.

The ability to redirect signals is achieved by 'bouncing' them off walls constructed from two barriers arranged either horizontally or vertically:

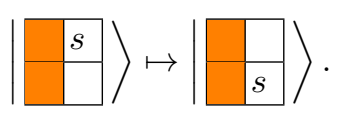

where $s$ again denotes the signal and the shaded cells denote the barriers which causes the signal to change direction. If there is only one barrier present in the 
four cell square being operated on then the signal simply propagates as normal and is not deflected:

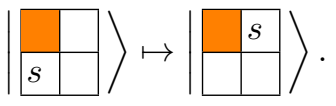

Using only these basic rules of signal propagation and signal reflection from barrier walls, signal delay (Fig. 3) and signal swapping (Fig. 4) tiles can be constructed. All of the rules presented so far are permutations of some of the base elements of the vector space generated by

$$
\left\{\left|\begin{array}{|l|l}
\hline w & x \\
\hline y & z
\end{array}\right\rangle\right\}_{w, x, y, z \in\{\epsilon, 0,1, \boldsymbol{\square}}
$$

therefore $U$ is indeed unitary on the subspace upon which its action has so far been described.

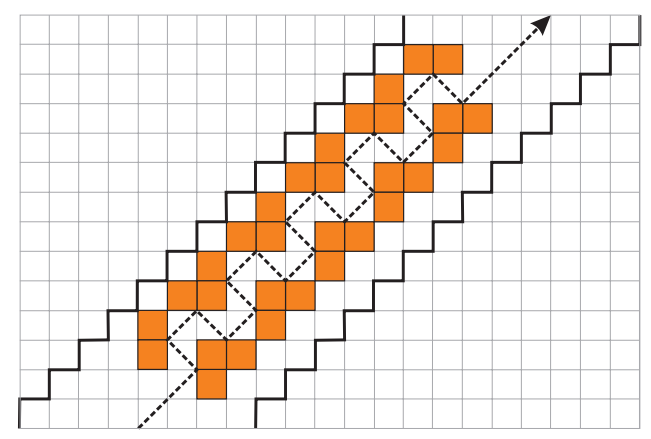

Fig. 3. The 'identity circuit' tile, an $8 \times 14$ tile taking 24 time-steps, made by repeatedly bouncing the signal from walls to slow its movement through the tile. The dotted line gives the signal trajectory, with the arrow showing the exit point and direction of signal propagation. The bold lines show the tile boundary.

\subsection{Gates}

To allow a universal set of gates to be implemented by the PQCA, certain combinations of signals and barriers can be assigned special importance. The Hadamard operation on a single qubit-carrying signal can be implemented by interpreting a signal passing through a diagonally oriented wall, analogous to a semitransparent barrier in physics. This has the action defined by the following rule:

$$
\begin{aligned}
& \left.\begin{array}{|l|l|l|l|l|l|}
\hline & \\
\hline 0 &
\end{array}\right\rangle \frac{1}{\sqrt{2}}\left|\begin{array}{l}
\hline \\
\hline
\end{array}\right\rangle+\frac{1}{\sqrt{2}} \mid \begin{array}{l}
\hline \\
\hline
\end{array}
\end{aligned}
$$

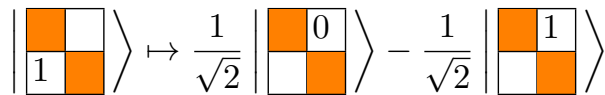




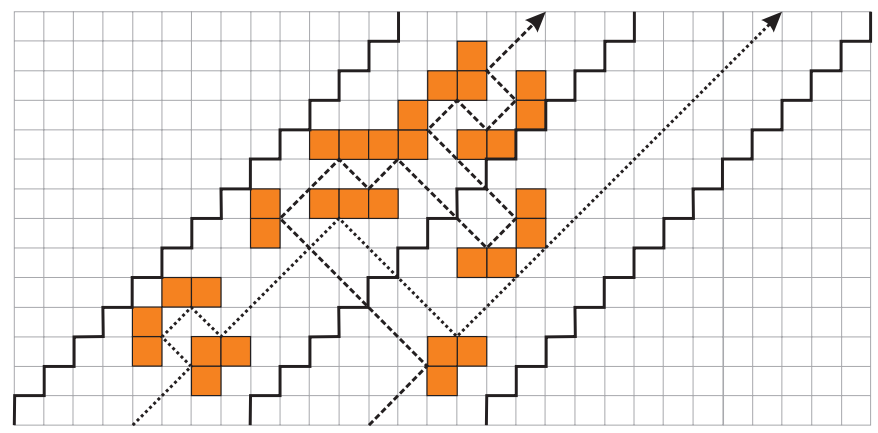

Fig. 4. The 'swap circuit' tile, a $16 \times 14$ tile, where both input signals are permuted and exit synchronously after 24 time-steps. As the first signal (bottom left) is initially delayed, there is no interaction.

This implements the Hadamard operation, creating a superposition of configurations with appropriate phases. Using this construction a Hadamard tile can be constructed (Fig. 5) by simply adding a semitransparent barrier to the end of the previously defined delay (identity) tile (Fig. 3). A way of encoding two

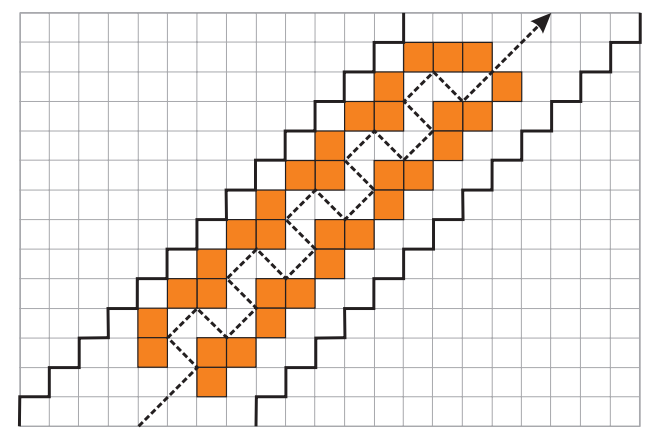

Fig. 5. The 'Hadamard gate' tile applies the Hadamard operation to the input signal. It is a modification of the identity circuit tile, with a diagonal (semitransparent) barrier added at the end which performs the Hadamard operation.

qubit gates in this system is to consider that two signals which cross paths interact with one another. The controlled-R $\left(\frac{\pi}{4}\right)$ operation can be implemented by considering signals that cross each other as interacting only if they are both 1 , in which case a global phase of $e^{\frac{i \pi}{4}}$ is applied. Otherwise the signals continue as normal. This behaviour is defined by the following rule:

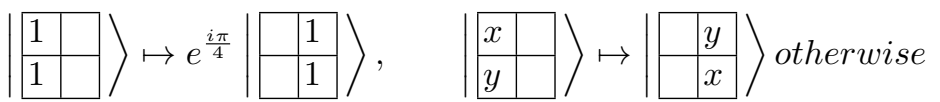


where $x, y \in\{0,1\}$. This signal interaction which induces a global phase change allows the definition of both a two signal controlled-R $\left(\frac{\pi}{4}\right)$ tile (Fig. 6) and a single signal $\mathrm{R}\left(\frac{\pi}{4}\right)$ operation tile (Fig. 7). These rules are simply a permutation

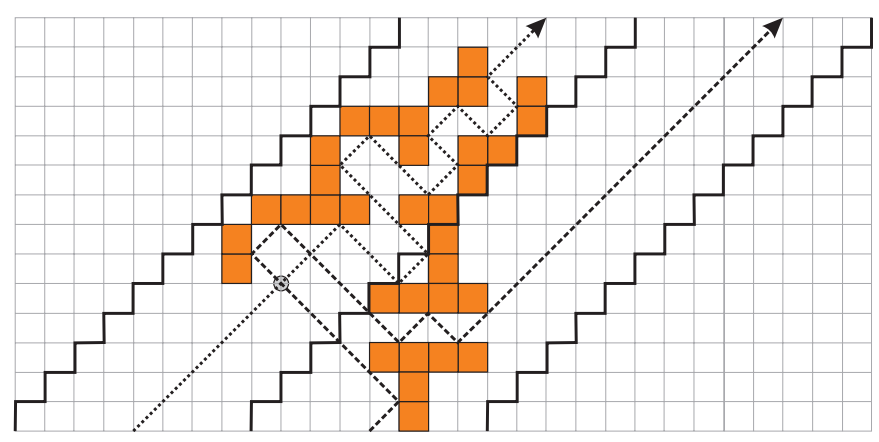

Fig. 6. The 'controlled-R $\left(\frac{\pi}{4}\right)$ gate' tile applies the controlled-R $\left(\frac{\pi}{4}\right)$ operation to the two input qubits, by causing the signals to interact at the highlighted point (grey circle). The qubits are then synchronised so that they exit at the same time along their original paths. No swapping takes place.

and phase change of base elements of the form:

$$
\left\{\mid \begin{array}{|l|l|}
\mid x & \\
\hline y & \rangle
\end{array}\right\}_{x, y \in\{0,1\}}
$$

(and their rotations), therefore $U$ is a unitary operation on the subspace upon which its action has so far been described. Wherever $U$ has not yet been defined, it is the identity. Hence $U$ is unitary.

\subsection{Circuits: Combining Gates}

A signal is given an $8 \times 14$ tile $(16 \times 14$ for two signal operations $)$ in which the action is encoded. The signals enter each tile at the fifth cell from the left, and propagate diagonally NE. Each time step finds the tile shifted one cell to the right to match this diagonal movement, giving a diagonal tile. The signal exits the tile 14 cells North and East of where it entered. This allows these tiles to be composed in parallel and sequentially with the only other requirement being that the signal exits at the appropriate point, i.e. the fifth cell along the tile, after 24 time-steps. This ensures that all signals are synchronised as in Fig. 2 (right), allowing larger circuits to be built from these elementary tiles by simply plugging them together. Non-contiguous gates can also be wired together using appropriate wall constructions to redirect and delay signals so that they are correctly synchronised. 


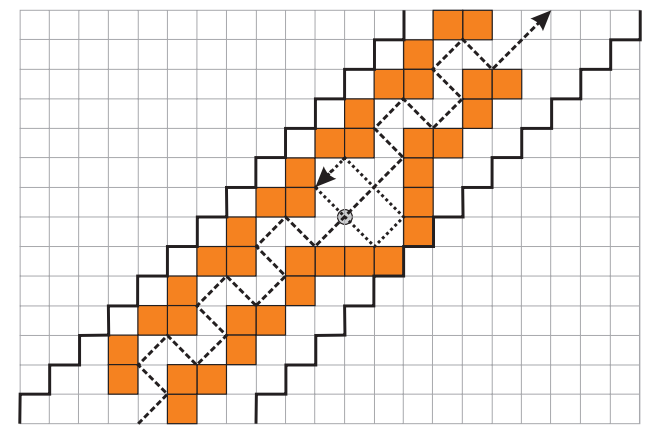

Fig. 7. The ' $R\left(\frac{\pi}{4}\right)$ gate' tile. This tile makes use of a signal, set to $|1\rangle$, which loops inside the grid every six time-steps, ensuring that it will interact with the signal that enters the tile, and causing it to act as the control qubit to a controlled-R $\left(\frac{\pi}{4}\right)$ operation. It therefore acts as a phase rotation on the input qubit, which passes directly through. After 24 time-steps the auxiliary control signal has returned to its origin, unchanged, hence the tile can be reused.

The implemented set of quantum gates, the identity, Hadamard, swap, $\mathrm{R}\left(\frac{\pi}{4}\right)$ and controlled-R $\left(\frac{\pi}{4}\right)$, gives a universal set. Indeed the standard set of CNOT, H, $\mathrm{R}\left(\frac{\pi}{4}\right)$ can be recovered as follows:

$$
\mathrm{CNoT}|\psi\rangle=(\mathbb{I} \otimes H)(\mathrm{CR}(\pi / 4))^{4}(\mathbb{I} \otimes H)|\psi\rangle
$$

where $\mathrm{CR}\left(\frac{\pi}{4}\right)^{4}$ denotes four applications of the controlled-R $\left(\frac{\pi}{4}\right)$ gate, giving the controlled-PHASE operation.

\section{Conclusion}

This paper presents a simple PQCA which is capable of simulating all other PQCA, preserving the topology of the simulated PQCA. This means that the initial configuration and the forward evolution of any PQCA can be encoded within the initial configuration of this PQCA, with each simulated cell encoded as a group of adjacent cells in the PQCA, i.e. intrinsic simulation. The construction in section 2 is given in two-dimensions, which can be seen to generalise to $n>1$ dimensions. The main, formal result of this work can therefore be stated as:

Claim 1 There exists an n-dimensional $U$-defined $P Q C A, G$, which is an intrinsically universal $P Q C A$. Let $H$ be a n-dimensional $V$-defined $P Q C A$ such that $V$ can be expressed as a quantum circuit $C$ made of gates from the set HADAMARD, CNOT, and $\mathrm{R}\left(\frac{\pi}{4}\right)$. Then $G$ is able to intrinsically simulate $H$.

Any finite-dimensional unitary $V$ can always be approximated by a circuit $C(V)$ with an arbitrary small error $\varepsilon=\max _{|\psi\rangle}|| V|\psi\rangle-C|\psi\rangle \|$. Assuming instead that $G$ simulates the $C(V)$-defined PQCA, for a region of $s$ cells over a 
period $t$, the error with respect to the $V$-defined PQCA will be bounded by $s t \varepsilon$. This is due to the general statement that errors in quantum circuits increase, at most, proportionally with time and space [25]. Combined with the fact that PQCA are universal [5]6, this means that $G$ is intrinsically universal, up to this unavoidable approximation.

\subsection{Discussion and Future Work}

QC research has so far focused on applications for more secure and efficient computing, with theoretical physics supporting this work in theoretical computer science. The results of this interdisciplinary exchange led to the assumptions underlying computer science being revisited, with information theory and complexity theory, for example, being reconsidered and redeveloped. However, information theory also plays a crucial role in the foundations of theoretical physics. These developments are also of interest in theoretical physics studies where physical aspects such as particles and matter are considered; computer science studies tend to consider only abstract mathematical quantities. Universality, among the many computer science concepts, is a simplifying methodology in this respect. For example, if the problem being studied crucially involves some idea of interaction, universality makes it possible cast it in terms of information exchanges together with some universal information processing. This paper presents an attempt to export universality as a tool for application in theoretical physics, a small step towards the goal of finding and understanding a universal physical phenomenon, within some simplified mechanics. Similar to the importance of the idea of the spatial arrangement of interactions in physics, intrinsic universality has broader applicability than computation universality and must be preferred. In short, if only one physical phenomenon is considered, it should be an intrinsically universal physical phenomenon, as it could be used to simulate all others.

The PQCA cell dimension of the simple intrinsically universal construction given here is four (empty, a qubit $(|0\rangle$ or $|1\rangle)$, or a barrier). In comparison, the simplest classical Partitioned CA has cell dimension two [19]. Hence, although the intrinsically universal PQCA presented here is the simplest found, it is not minimal. In fact, one can also manage [7] an intrinsically universal PQCA with a cell dimension of three, in two different ways. One way is to encode the spin degree of freedom (0 and 1) into a spatial degree of freedom, so that now the semitransparent barrier either splits or combines signals. The second way is to code barriers as pairs of signals as in the Billiard Ball CA model [19. These constructions may be minimal, but are not as elegant as the one presented here. In future work we will show that there is a simple, greater than two-dimensional PQCA which is minimal, as it has a cell dimension of two. 


\section{Acknowledgements}

The authors would like to thank Jérôme Durand-Lose, Jarkko Kari, Jacques Mazoyer, Kenichi Morita, Nicolas Ollinger, Guillaume Theyssier and Philippe Jorrand.

\section{References}

1. Albert, J., Culik, K.: A simple universal cellular automaton and its one-way and totalistic version. Complex Systems 1 (1987) 1-16

2. Arrighi, P.: Algebraic characterizations of unitary linear quantum cellular automata. In: Proceedings of MFCS, Lecture Notes in Computer Science. Volume 4162., Springer (2006) 122

3. Arrighi, P., Fargetton, R.: Intrinsically universal one-dimensional quantum cellular automata. In: Proceedings of the Development of Computational Models workshop (DCM '07). (2007)

4. Arrighi, P., Fargetton, R., Wang, Z.: Intrinsically universal one-dimensional quantum cellular automata in two flavours. Fundamenta Informaticae 21 (2009) 10011035

5. Arrighi, P., Grattage, J.: Intrinsically universal $n$-dimensional quantum cellular automata. Extended version of this paper. ArXiv preprint: arXiv:0907.3827 (2009)

6. Arrighi, P., Grattage, J.: Partitioned quantum cellular automata are intrinsically universal. Submitted (2009)

7. Arrighi, P., Grattage, J.: Two minimal $n$-dimensional intrinsically universal quantum cellular automata. Manuscript (2009)

8. Arrighi, P., Nesme, V., Werner, R.: Unitarity plus causality implies localizability. Quantum Information Processing (QIP) 2010, ArXiv preprint: arXiv:0711.3975 (2007)

9. Arrighi, P., Nesme, V., Werner, R.F.: Quantum cellular automata over finite, unbounded configurations. In: Proceedings of MFCS, Lecture Notes in Computer Science. Volume 5196., Springer (2008) 64-75

10. Banks, E.R.: Universality in cellular automata. In: Proceedings of the 11th Annual Symposium on Switching and Automata Theory (SWAT '70), Washington, DC, USA, IEEE Computer Society (1970) 194-215

11. Brennen, G.K., Williams, J.E.: Entanglement dynamics in one-dimensional quantum cellular automata. Phys. Rev. A 68(4) (Oct 2003) 042311

12. Durand, B., Roka, Z.: The Game of Life: universality revisited, Research Report 98-01. Technical report, Ecole Normale Suprieure de Lyon (1998)

13. Durand-Lose, J.O.: Reversible cellular automaton able to simulate any other reversible one using partitioning automata. In: Latin '95, number 911, Lecture Notes in Computer Science, Springer (1995) 23024-4

14. Durand-Lose, J.O.: Intrinsic universality of a 1-dimensional reversible cellular automaton. In: Proceedings of STACS '97, Lecture Notes in Computer Science, Springer (1997) 439

15. Durr, C., Le Thanh, H., Santha, M.: A decision procedure for well-formed linear quantum cellular automata. In: Proceedings of STACS '96, Lecture Notes in Computer Science, Springer (1996) 281-292

16. Feynman, R.P.: Quantum mechanical computers. Foundations of Physics (Historical Archive) 16(6) (1986) 507-531 
17. Lloyd, S.: A theory of quantum gravity based on quantum computation. ArXiv preprint: quant-ph/0501135 (2005)

18. Margolus, N.: Physics-like models of computation. Physica D: Nonlinear Phenomena 10(1-2) (1984)

19. Margolus, N.: Parallel quantum computation. In: Complexity, Entropy, and the Physics of Information: The Proceedings of the 1988 Workshop on Complexity, Entropy, and the Physics of Information, May-June 1989, Santa Fe, New Mexico, Perseus Books (1990) 273

20. Mazoyer, J., Rapaport, I.: Inducing an order on cellular automata by a grouping operation. In: Proceedings of STACS '98, Lecture Notes in Computer Science, Springer (1998) 116-127

21. Morita, K.: Reversible simulation of one-dimensional irreversible cellular automata. Theoretical Computer Science 148(1) (1995) 157-163

22. Morita, K., Harao, M.: Computation universality of one-dimensional reversible (injective) cellular automata. IEICE Trans. Inf. \& Syst., E 72 (1989) 758-762

23. Morita, K., Ueno, S.: Computation-universal models of two-dimensional 16-state reversible cellular automata. IEICE Trans. Inf. \& Syst., E 75 (1992) 141-147

24. Nagaj, D., Wocjan, P.: Hamiltonian Quantum Cellular Automata in 1D. ArXiv preprint: arXiv:0802.0886 (2008)

25. Nielsen, M.A., Chuang, I.L.: Quantum Computation and Quantum Information. Cambridge University Press (October 2000)

26. Ollinger, N.: Universalities in cellular automata a (short) survey. In Durand, B., ed.: First Symposium on Cellular Automata "Journées Automates Cellulaires" (JAC 2008), Uzès, France, April 21-25, 2008. Proceedings, MCCME Publishing House, Moscow (2008) 102-118

27. Ollinger, N., Richard, G.: A Particular Universal Cellular Automaton. In Neary, T., Woods, D., Seda, A.K., Murphy, N., eds.: CSP, Cork University Press (2008) 267-278

28. Pérez-Delgado, C., Cheung, D.: Local unitary quantum cellular automata. Physical Review A 76(3) (2007) 32320

29. Raussendorf, R.: Quantum cellular automaton for universal quantum computation. Phys. Rev. A 72(022301) (2005)

30. Schumacher, B., Werner, R.: Reversible quantum cellular automata. ArXiv preprint quant-ph/0405174 (2004)

31. Shepherd, D.J., Franz, T., Werner, R.F.: A universally programmable quantum cellular automata. Phys. Rev. Lett. 97(020502) (2006)

32. Theyssier, G.: Captive cellular automata. In: Proceedings of MFCS 2004, Lecture Notes in Computer Science, Springer (2004) 427-438

33. Toffoli, T.: Computation and construction universality of reversible cellular automata. J. of Computer and System Sciences 15(2) (1977)

34. Van Dam, W.: Quantum cellular automata. Masters thesis, University of Nijmegen, The Netherlands (1996)

35. Vollbrecht, K.G.H., Cirac, J.I.: Reversible universal quantum computation within translation-invariant systems. New J. Phys Rev A 73 (2004) 012324

36. von Neumann, J.: Theory of Self-Reproducing Automata. University of Illinois Press, Champaign, IL, USA (1966)

37. Watrous, J.: On one-dimensional quantum cellular automata. Complex Systems 5(1) (1991) 19-30

38. Watrous, J.: On one-dimensional quantum cellular automata. In: Proceedings of the 36th IEEE Symposium on Foundations of Computer Science, Washington, DC, USA, IEEE Computer Society (1995) 528-537 